\title{
The behavioural phenotype of Smith-Magenis syndrome: Evidence for a gene-environment interaction.
}

\author{
Taylor, L. and Oliver, C. \\ Cerebra Centre for Neurodevelopmental Disorders, \\ School of Psychology, \\ University of Birmingham
}

Please use this reference when citing this work:

Taylor, L. and Oliver, C. (2008). The behavioural phenotype of Smith-Magenis syndrome: Evidence for a gene-environment interaction. Journal of Intellectual Disability Research, 52, 830-841. (DOI: 10.1111/j.1365-2788.2008.01066.x)

The Cerebra Centre for Neurodevelopmental Disorders,

School of Psychology, University of Birmingham, Edgbaston, Birmingham, B15 2TT

Website: www.cndd.Bham.ac.uk

E-mail: cndd-enquiries@contacts.bham.ac.uk 


\begin{abstract}
Background

Behaviour problems and a preference for adult contact are reported to be prominent in the phenotype of Smith-Magenis syndrome. In this study we examined the relationship between social interactions and self-injurious and aggressiveldisruptive behaviour in SmithMagenis syndrome to explore potential operant reinforcement of problem behaviours and thus a gene-environment interaction.
\end{abstract}

\title{
Method
}

Observational data on five children with Smith-Magenis syndrome (age range 3 to 13 years) were collected for between 9 and 12 hours. The associations between purported phenotypic behaviours and two environmental events (adult attention and demands) were examined using descriptive analysis.

\section{Results}

All participants engaged in self-injurious behaviour and aggressive/disruptive outbursts. Sequential analyses of aggressive/disruptive outbursts and self-injury revealed that these behaviours were evoked by low levels of adult attention and led to increased levels of attention following the behaviours in three and two participants respectively out of the four for whom this analysis was possible.

\section{Conclusion}

Problem behaviour in Smith-Magenis syndrome was evoked by decreased social contact in three out of four children. These data, considered alongside the preference for adult contact and the significantly increased prevalence of these behaviours in Smith-Magenis syndrome, illustrate a potential gene-environment interaction for problem behaviour in this syndrome. 


\section{Introduction}

Smith-Magenis syndrome is caused by a deletion on chromosome 17 (17p11.2) (Juyal et al., 1996) and occurs in approximately 1 in 25,000 births (Greenberg et al., 1991). Facial features include a flat, broad head and prominent forehead, heavy brows, up-slanting eyes, depressed nasal bridge and a wide mouth with fleshy and inverted central portion of the upper lip. Other features include short and broad hands, short stature, a hoarse deep voice, speech delay and hearing loss (Greenberg et al., 1991). The majority of individuals with Smith-Magenis syndrome evidence developmental delay and moderate to severe intellectual disability (Dykens, Finucane, \& Gayley, 1997; Moncla et al., 1991; Udwin, Webber, \& Horn, 2001).

Studies suggest that Smith-Magenis syndrome is associated with a behavioural phenotype (Allanson, Greenberg, \& Smith, 1999), as defined by an increased probability of behavioural characteristics compared to individuals without the syndrome (Dykens, 1995). Most notably self-injurious behaviour is frequently reported in the forms of hand biting, self pinching or scratching, picking at sores, hitting the head or body, and tearing or picking finger/toenails or the skin around the nails and inserting objects into bodily orifices (Colley, Leversha, Voullaire, \& Rogers 1990; Crumley, 1998; Finucane, Kurtz, Babu, \& Scott 1993; King et al., 2004; Lockwood et al., 1988; Sarimski 2004; Smith et al., 1986; Stratton et al., 1986; Arron, Oliver, Berg et al., In review). Other commonly reported behaviours include aggression, frequent 'temper tantrums', hyperactivity, restlessness, distractibility, and some autistic features, such as dislike of transitional periods (Oliver, Berg, Burbidge et al., In review). Some behaviour are described as 'unique' to Smith-Magenis syndrome; 'selfhugging,' 'hand squeezing' (Finucane, Konar, Haas-Givler, Kurtz, \& Scott 1994; Willekens, Cock, \& Fryns 2000), hand licking and page flipping (Dykens, Finucane, \& Gayley 1997 and see Shelley, Robertson and Turk, 2007; Moss, Oliver, Arron et al. In review). Sleep disturbance is well documented in Smith-Magenis syndrome (Colley, Leversha, Voullaire, \& Rogers 1990; De Leersnyder et al., 1999; 2001a, b; 2003; Greenberg, et al., 1991; Potocki, Shaw, Stankicwicz, \& Lupski 2003) and includes difficulties falling asleep at night, shortened sleep cycles, frequent and prolonged night waking and early morning waking (Allanson, Greenberg, \& Smith, 1999; McElwee \& Bernard, 2002; Moncla et al., 1991; Smith, Dykens, \& Greenberg, 1998b). 
Estimates of self-injurious behaviour in Smith-Magenis syndrome are consistent, with studies reporting high prevalence figures e.g. 92\% (Dykens \& Smith, 1998), 96.6\% (Finucane et al., 2001), 98\% Arron et al., In review). It has been assumed that self-injurious behaviour in Smith-Magenis syndrome has strong biological determinants with support for this proposal gleaned from the comparatively high prevalence and observed high pain thresholds (Greenberg et al., 1991). However, several anecdotal reports suggest self-injury may be associated with interpersonal events, particularly decreases in adult attention or increases in demand (Dykens, Finucane, \& Gayley, 1997; Haas-Givler, 1994; King et al., 2004). In combination with the unusually high prevalence data this indicates that biological factors might interact with interpersonal events to evoke episodes of problem behaviour.

There is a substantial body of empirical evidence suggesting that the maintenance of selfinjurious and aggressive/disruptive behaviours can be accounted for by the application of the principles of learning theory to the observed relationships between self-injury and interpersonal events (Carr \& Durand, 1985; Iwata et al., 1982; Oliver, 1995). In syndromes such as Cornelia de Lange and Lesch-Nyhan, in which self-injurious behaviour is common (Christie et al., 1982; Hyman, Oliver, \& Hall, 2002; Sloneem et al., In review), the importance of the association between the social environment and self-injurious behaviour has been demonstrated (Hall, Oliver, \& Murphy, 2001b, Arron et al., 2006; Moss et al., 2005). Research into Rett syndrome has also shown that there is an interrelationship between interpersonal events and self-injurious behaviour (Oliver, Murphy, Crayton, \& Corbett, 1993). Operant reinforcement of problem behaviour has not been explored in Smith-Magenis syndrome and it is clear that there is a case for extending research into interactions between phenotypic characteristics and operant reinforcement.

Anecdotal descriptions of the 'personality' of individuals with Smith-Magenis syndrome note fluctuations between extreme positive and negative mood. Descriptions of babies with Smith-Magenis syndrome vary from 'placid' (Colley, Leversha, Voullaire, \& Rogers, 1990), 'perfect', tranquil (Moncla et al., 1991), 'happy' babies (Smith, et al., 1998), to 'sad, insecure emotional' babies (Moncla et al., 1991). Later developing friendly, outgoing, 'eager to please' characteristics alongside a 'well developed sense of humour' (Smith, Dykens, \& Greenberg, 1998a) are consistently accompanied by 'frustration and unhappiness' (Colley, Leversha, Voullaire, \& Rogers, 1990), hyperactivity, excessive aggression, self-stimulatory activity, self-injurious behaviour, 'temper tantrums,' and 
repetitive questioning (Dykens \& Smith, 1998; Finucane, Dirrigl, \& Simon, 2001; Greenberg et al., 1991; Madduri, Turcich, Lupski, \& Potocki, 2002; Smith, Dykens, \& Greenberg, 1998a; Oliver et al., In review). Individuals with Smith-Magenis syndrome are noted to have a preference for adult contact and, should such contact be denied, physical and verbal aggressive outbursts towards others (and furnishings) and self-injurious behaviour frequently follow (Haas-Givler, 1994; Haas-Givler \& Finucane, 1995; Spencer, 2003; Willekens, Cock, \& Fryns, 2000). These reports lead to the hypothesis that problem behaviours associated with Smith-Magenis syndrome could be influenced by an interaction between a genetically predisposed strong preference for adult contact and operant reinforcement of challenging behaviours by the presentation of social contact contingent on such behaviour. This hypothesis can be addressed by functional assessment of problem behaviours in Smith-Magenis syndrome

The literature regarding the functional assessment of self-injury and aggression to identify possible relationships between these behaviours and interpersonal events is growing. A number of studies have demonstrated a functional relationship between behaviour and social/environmental factors using both experimental analyses and lag sequential methods applied to naturally occurring streams of behaviour (Carr \& Durand, 1985; Hall, Oliver, \& Murphy, 2001a; Iwata et al., 1982; Oliver, Hall, \& Murphy, 2005) In this study we conduct objective observations of the behaviour of children with Smith-Magenis syndrome to 1) quantify levels of problem behaviour in Smith-Magenis syndrome and 2) examine the association between problem behaviour in Smith-Magenis syndrome and environmental events indicative of social reinforcement processes using lag sequential methods.

\section{Method}

\section{Participants}

Participants were recruited through the UK parent-professional support group (The SmithMagenis Syndrome Foundation) and also during a Smith-Magenis Syndrome Foundation Conference/Family Day. The inclusion criteria were that participants had a diagnosis of Smith-Magenis syndrome caused by a deletion of chromosome $17 \mathrm{p} 11.2$, were aged between 3-18 years old, and attended a school within 150 miles of the project base. 
Six individuals were considered for inclusion. However, before the study began one of these individual was excluded from school. Consequently, five individuals (two females and three males) participated. Demographic information was collated from parents by interview and with the completion of the Vineland Adaptive Behavior Scales (Sparrow, Balla, \& Cicchetti, 1984). Participant demographics and prescribed medication are presented in Table 1. The age of the participants ranged from 3 to 13 years. The developmental ages of the participants (calculated from the Vineland Adaptive Behavior Scale; Sparrow, Balla, \& Cicchetti, 1984), ranged from 1.7 years to 4.9 years. Participants 1 to 4 had moderate deficits, and participant 5 had a severe deficit. Differences in standard scores across the three Vineland subscales approaching 1 standard deviation (15) are notable and all participants scored higher in socialisation than communication and daily living skills. This pattern is markedly dissimilar to that seen in autistic spectrum disorder (Carter et al., 1998). Two of the participants (P1 and P2) attended schools for children with intellectual disability, two participants attended schools for children with autistic characteristics (P4 and P5) and one participant attended a mainstream school (P3). It is notable that all participants received one-to-one support from an adult, from the time they arrived in school, until they left school at the end of the school day.

\section{+++++++++++++ Insert Table 1 here ++++++++++++++}

\section{Procedure}

Ethical review was obtained from the University's ethics committee and assent for participation was gained from parents because the children were not able to give informed consent. All participants were observed in their school environments during break-times, classroom teaching, including group and single one-to-one sessions, and outside classroom sessions. Observation periods ranged from 9.3 to 12.4 hours. All participants were observed between the hours of $9.00 \mathrm{am}$ to $3.15 \mathrm{pm}$. The observers endeavoured to remain as inconspicuous as possible and did not interact with the child being observed. The observer requested that teachers and carers interacted with the child in their usual manner.

Observations were stopped at anytime there was the potential for inappropriate intrusion into the child's privacy (for example, during toileting). The behaviour of participants and adults were recorded on a Hewlett Packard Jornada model 720 palm-held computer using the ObsWin program (Martin, Oliver, \& Hall, 2001), which allows continuous recording of 
the frequency and duration of participant behaviours and environmental events by recording onsets and offsets of each behaviour.

\section{Observational response definitions}

Environmental events that were recorded included attention (any verbal or physical contact made by the caregiver towards the participant that did not include a denial or demand) and demand (any verbal or physical direction by the caregiver to the participant to initiate or complete an action or a task). Composite variables were formed for self-injurious behaviour and aggressive/disruptive outbursts. Participants' behaviours included the following: selfinjurious behaviour (any occurrences of head-banging, self-slapping, biting self, nail and skin picking, poke eye and/or inserting fingers up nose) and aggressive/disruptive outburst (any occurrence of kicking, push furniture away, banging of objects, striking others and/or spitting). Definitions were devised on the basis of a literature review of the behaviour of individuals with Smith-Magenis syndrome.

\section{Inter observer reliability}

Inter-observer reliability was calculated for approximately $10 \%$ (5 hours) of the total observation time (55 hours) across three participants. Reliability observation periods were conducted live. In order to control for 'chance' levels of agreement, Cohen's Kappa (Cohen, 1960) was calculated for each target variable under observation. Agreement was calculated based on 10 second bins for the presence of each target variable (Oliver, Hall, \& Nixon, 1999).

The mean Kappa values for environmental events were: Attention .75 (range $=.00$ to 1.00 ) and demand .62 (range $=-.2$ to 1.00$)$. Very low Kappa values were obtained only when the frequency of behaviours was very low. The mean Kappa value for variables employed across individuals was .6 (range .2 to .86). Kappa values of .4 to .6 may be regarded as 'fair', .60 to .75 as 'good' and values of '.75 or so' can signify excellent agreement (Fleiss, 1981). The mean inter-observer agreement across environmental events may therefore be considered 'good.' The mean Kappa statistic across behaviours displayed by participants fell within the 'fair' range with two below the 'fair' range, ten within the 'fair' range, twelve within the 'good' range and two within the 'excellent' range. 


\section{Data analysis}

\section{Summary statistics}

The percentages of time that the environmental events and participant behaviours occurred were calculated using the OBSWIN software.

\section{Co-occurrence analysis}

To evaluate the association between participants' behaviours and environmental events, datasets were analysed on the basis of 10s windows employing Yule's Q to describe the magnitude of an association between a condition and behaviours. Yule's Q values were calculated employing the ObsWin statistical analysis package (Martin, Oliver, \& Hall, 2001). Yule's $Q$ is calculated on the basis of a $2 \times 2$ matrix, with resultant values ranging from -1 to +1 and is an indication of the magnitude of difference between the conditional and unconditional probability of target behaviour occurring. For this analysis, any behaviour had to occur in a10s windows, otherwise it was deemed insufficient for statistical analysis. A Yule's Q value of .3 or higher was deemed to indicate the presence of a significant association. This value of .3 can be interpreted as indicating that the surplus of consistent pairings of behaviours and environmental events over inconsistent pairings is $30 \%$ of all non-tied data pairs. A Yule's Q value of 0 indicates a null relationship between the response categories (Yoder \& Feurer, 2000). The identification of a potential social function of behaviour is based on the increased likelihood of a behaviour occurring (such as self-injurious behaviour and aggressive/disruptive outbursts) with environmental events typically reported in the literature to be social antecedents for problem behaviour (such as low levels of adult attention and presence of demands) (Carr \& Durand, 1985; Iwata et al., 1982).

\section{Sequential analysis}

Analysis of the sequential relationships between participant and environmental condition was analysed using ObsWin statistical analysis package (Martin, Oliver, \& Hall, 2001) employing time based lag sequential analysis (Bakeman \& Gottman, 1997). Sequential relationships were analysed for each participant by calculating the probability of an environmental event e.g. adult attention, being present within 10 s intervals of the onset of a response category e.g. self-injury, for 120 s before and after each behaviour. The degree of association between two response categories at each lag is indicated by a positive or negative Yule's Q value and .3 (+/-) was deemed to indicate a significant association. 
Periods of $10 \mathrm{~s}$ windows preceding and following the onset of each of the behaviours were employed with an additional procedure for terminating calculations when another onset of behaviour was identified within 120s preceding or following the index behaviour.

\section{Results}

\section{Duration of problem behaviours}

To fulfil the first aim of the study the percentage duration for participant problem behaviours were calculated and are presented in Table 2. All participants were observed engaging in Self-injurious Behaviour (range 1.07\% to 10.26\%) and this was higher for P1, P2 and P5 than Aggressive/Disruptive Outbursts (range .17\% to 5.81\%). The two participants observed to engage more in Aggressive/Disruptive Outbursts were the older males $\mathrm{P} 3$ and $\mathrm{P} 4$. The mean percentage of adult attention across participants was $47.7 \%$ (range $15.7 \%$ to $75.4 \%$ ) and the mean percentage of adult demands across participants was $15.9 \%$ (range from $2.2 \%$ to $45.8 \%$ ).

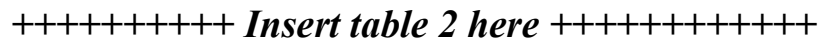

\section{Co-occurrence of problem behaviours and environmental events}

To fulfil the second aim of the study and examine the association between problem behaviour and environmental events Yule's Q values were calculated. The results for the analysis of co-occurrence for problem behaviours and environmental events are presented in Table 3. Results revealed a significant negative association between Self-Injurious Behaviour and Adult Attention for P4 (Yule's Q = -.52) and P5 (Yule's Q = -.44). Similarly there was a significant negative association for both Self-Injurious Behaviour and Adult Demand for P1 (Yule's Q = -.33) and P5 (Yule's Q = -.84). There was a significant negative association between Aggressive/Disruptive Outbursts and Adult Attention for P3 (Yule's $\mathrm{Q}=-.31$ ) and $\mathrm{P} 4$ (Yule's $\mathrm{Q}=-.52$ ). For P5, a significant positive association with Aggressive/Disruptive Outbursts and Attention (Yule's Q = .47) was evident. In summary, results indicated that for three participants Self-injurious Behaviour (P1, P4, and P5) and Aggressive/Disruptive Behaviour (P3 and P4) were significantly associated with low levels of Adult Attention. 


\section{Sequential analysis}

Analysis of co-occurrence does not allow examination of sequence and thus whether environmental events precede or follow participant's behaviours. Therefore, a sequential analysis for Self-Injurious Behaviour and Aggressive/Disruptive Outbursts with Adult Attention and Demand for P1, P3, P4 and P5 who evidenced a Yule's Q above .3 (+/-) indicating an association between participant behaviour and environmental events was conducted. The results of the lag sequential analysis are shown in Figure 1.

\section{++++++++++++ Insert figure 1 here ++++++++++++++}

Figure 1 shows the unconditional and conditional probability of the environmental events from lag -12 (120s. preceding the behaviour) to lag +12 (120s. following the behaviour) given the onset of Self-Injurious Behaviour or Aggressive Disruptive Behaviour. The shaded area on the graphs indicates the period within which the conditional probability of the environmental event is significantly different (Yule's $Q>.3$ ) from the unconditional probability. Thus, outside the shaded area differences between the conditional and unconditional probabilities can be considered comparable.

For P4 and P5 the onset of Self-Injurious Behaviour is associated with a decline in the probability of Adult Attention, with the level of Adult Attention steadily falling in the period preceding Self-Injurious Behaviour and rising again following onset. For P4, the conditional probability of Adult Attention shows a slight decrease ten seconds before the onset of Self-Injurious Behaviour (Yule's $Q=-.52$ ). Following onset, the probability of Adult Attention remains low but increases 60 seconds later, approaching its unconditional probability. For P5, the conditional probability of Adult Attention shows a significant decrease 20 seconds before the onset of Self-Injurious Behaviour (Yule's Q = -.44). Following onset, the probability of Adult Attention increases immediately, drops for 20 seconds and increases 50 seconds later again almost reaching the unconditional probability.

For P1 and P5, the onset of Self-Injurious Behaviour is associated with a decline in the probability of Adult Demand, with the level of Adult Demand slightly falling in the period preceding the Self-Injurious Behaviour and rising again following onset. For P1, the conditional probability of Adult Demand shows a small decrease ten seconds before the onset of Self-Injurious Behaviour (Yule's Q = -.33). Following onset, the probability of 
Adult Demand immediately increase for 20 seconds, drops for 40 seconds then gradually increases toward its unconditional probability of Adult Demand. For P5, the conditional probability of Adult Demand shows a small decrease 20 seconds before the onset of SelfInjurious Behaviour (Yule's Q = -.84). Following onset, the probability of Adult Demand increases 10 seconds later and rises toward its unconditional probability.

The pattern of decreasing levels of attention preceding SIB followed by increases in attention subsequent to SIB is also seen in Aggressive/Disruptive Outbursts for P3 and P4. For P3, the conditional probability of Adult Attention shows a decrease 20 seconds before the onset of Aggressive/Disruptive Outbursts (Yule's $\mathrm{Q}=-.31$ ). Following onset, the probability of Adult Attention increases 30 seconds later and approaches its unconditional probability. Similarly for P4, the conditional probability of Adult Attention shows a decrease 10 seconds before onset (Yule's $Q=-.52$ ). Following onset the probability of Adult Attention increases to above its unconditional probability. For P5 the profile is different, the conditional probability of Adult Attention is above the unconditional probability of Adult Attention prior to onset of the Aggressive/Disruptive Outbursts.

In summary, in four out of five participants there is a pattern of reduced levels of Adult Attention or Adult Demand preceding the onset of the Self-Injurious Behaviour and/or Aggressive/Disruptive Outbursts, with an increase in the probability of Adult Attention and or Adult Demand in the period following onset. There is a striking similarity between the profiles evidenced for self injury for P4 and P5 for self-injury and for P3 and P4 for aggressive and disruptive outbursts.

\section{Discussion}

This is the first study to employ objective observational analyses to describe the problem behaviours noted as prevalent in Smith-Magenis syndrome. The descriptive analysis of the social determinants of behaviour disorder is unique in the study of the causes of behaviour disorder in this syndrome. Operational definitions were employed and adequate levels of inter-rater reliability were achieved for the majority of behaviours. The natural environment in which observations were recorded ensured that any association between learned behaviours and social events was evaluated in the most appropriate setting. These methodological aspects of the study protect against threats to the validity of the findings. With regard to the importance of the findings, it is notable that all participants received 
one-to-one adult support in school and this reflects the level of behavioural support required by the children and thus the clinical significance of the behaviours under examination.

The Vineland Adaptive Behavior Scale indicated that four of the five participants showed moderate deficits, which is consistent with previous reports of the intellectual abilities of individuals with Smith-Magenis syndrome (Dykens, Finucane, \& Gayley, 1997; Moncla et al., 1991; Udwin, Webber, \& Horn, 2001). The exception to this was participant five who had the lowest scores in all the Vineland domains. Considering her age (13 years) these results, based on parental reports, are concordant with the conclusions of Udwin, Webber and Horn (2001) that older individuals with Smith-Magenis syndrome emerge as more dependent on carers than might have been expected from their general level of functioning. Interestingly, differences were evident across the three Vineland domains. All participants scored higher in socialisation than communication and daily living skills and the differences approached one standard deviation for the standard scores. Although it was not possible to employ statistical analysis, these findings are different from the non significant Vineland domain differences previously reported. (Dykens, Finucane, \& Gayley, 1997). This could be a reflection of the small sample in this study the different age groups of the two studies. Dykens, Finucane and Gayley (1997) used an older sample of individuals aged between 14 to 51 years old.

All participants displayed self-injurious behaviour and aggressive/disruptive outbursts. These results confirm that these aspects of the purported behavioural phenotype are evident when empirical observations are conducted, even though the data were drawn from observations that were only conducted for between nine and twelve hours for a small sample. It is possible that the observation period was too short to draw reliable conclusions regarding the duration and occurrence of behaviours. However, the nine to twelve hours of observations were conducted over different activities and over two days, thus giving a reasonably representative sample and the focus of the study was more on the association with environmental events. Whilst the data confirm the presence of two purported phenotypic behaviours (self-injurious and aggressive and disruptive behaviour), it is clearly critical to employ a larger sample and a comparison group to determine if behaviours are significantly more common for this syndrome (Dykens, Finucane, \& Gayley, 1997; Sarimski, 2004; Smith, Dykens, \& Greenberg, 1998a; Willekens, Cock, \& Fryn, 2000). It is also possible that the small sample was unrepresentative or biased in some way and future 
replication with a larger sample is warranted to appraise the generalisability of the findings reported here.

Results from the analysis of the association between problem behaviours and adult attention and demands are consistent with a learning theory interpretation. That is, these behaviours do not always appear to be random occurrences but occur as a reaction to environmental events, most notably decreased levels of adult attention. This confirms several anecdotal reports that self-injury in this syndrome is associated with decreased levels of adult attention (Dykens, Finucane, \& Gayley, 1997; Haas-Givler, 1994; King et al., 2004). The profile of decreasing levels of attention precipitating problem behaviour for P3, P4 and P5 with the behaviour evoking higher levels of attention was strikingly uniform and comparable to that first described by Hall and Oliver (1998), as evidence of mutual social reinforcement, and identified by Oliver et al., (2005) as related to increases in self-injury over time. It is also notable that levels of attention from adults were high at all times (one to one support) thus these results allude to an enhanced sensitivity to a reduction in adult attention.

Generally, these results are also consistent with research into the self-injurious behaviour of children with other syndromes in which self-injury is common. Hall, Oliver, and Murphy (2001b) found that children with Lesch-Nyhan syndrome were more likely to self injure during periods of low social interaction, suggesting that their self-injury was influenced by environmental factors. Similarly, Moss et al. (2005) and Arron et al., (2006) have demonstrated an association between self-injury and environmental events in Cornelia de Lange syndrome, another syndrome noted to have a high prevalence of self-injury (Sloneem et al., In review). However, it is important to note that in this study we did not experimentally manipulate the antecedents or consequences of behaviours and that the analysis is essentially descriptive and does not appraise causality (Berry, 2005). Further research should employ experimental functional analysis to confirm the observed impact of low levels of attention on these behaviours in Smith-Magenis syndrome.

Overall, the results of this study suggest that there is a need for functional analytic studies to further understand the behaviour of children and adults with Smith-Magenis and other syndromes. The relationship between low levels of adult attention and self-injurious behaviour and aggressive/disruptive outbursts in Smith-Magenis syndrome highlights the 
potential utility of experimental studies to explore how attention might be used effectively in behaviour management. Considering the significant behaviour problems early in the childhood of individuals with Smith-Magenis syndrome, and the high prevalence rates of self-injurious behaviour and aggressive disruption, early intervention with individuals with Smith-Magenis syndrome would seem appropriate. Future work is needed based on a combination of natural observations with analogue conditions to validate the conclusions drawn from lag sequential analysis and also to provide empirical data about the early development of the behavioural phenotype of Smith-Magenis syndrome.

The results of this study add to a growing literature showing an association between environmental events and problem behaviour in syndromes and, given the well documented higher prevalence of problem behaviours in some syndromes, allude to gene-environment interactions that govern the presentation of these behaviours. More specifically, we propose that a genetic predisposition to experience social or other stimuli as significantly rewarding in some syndromes can act as an accelerator for the generic mutual social reinforcement paradigm that underpins the natural development of problem behaviours (see Oliver, 1993, 1995; Oliver, et al., 2005, 2007). Additionally, the response cost of behaviours such as selfinjury in Smith-Magenis syndrome may be affected by reduced pain perception, thus the behaviour is comparatively unchecked. Future research should focus on the implications of the interaction between syndrome related enhanced motivation for social contact, impaired pain perception and mutual social reinforcement for prevention and early intervention. 


\section{References}

Allanson, J., Greenberg, F., \& Smith, A. (1999). The face of Smith-Magenis Syndrome: a subjective and objective study. Journal of Medical Genetics, 36, 394-397.

Arron, K., Oliver, C., Berg, K., Moss, J. and Burbidge, C. (In review). Delineation of behavioural phenotypes in genetic syndromes. 2. Prevalence, phenomenology and correlates of self-injurious and aggressive behaviour. British Journal of Psychiatry

Arron, K., Oliver, C., Hall, S., Sloneem, J., Forman, D., \& McClintock, K. (2006). Effects of Social Context on Social Interaction and Self-Injurious Behavior in Cornelia de Lange Syndrome. American Journal on Mental Retardation, 111, 184-192.

Bakeman, R., \& Gottman, J. (1997). Observing interaction: An introduction to sequential analysis (second edition). Cambridge University Press.

Berry, A. (2005). Observational studies identify associations, not causality. Anesth Analg, $101,1238-1241$.

Carter, A., Volkmar, F., Sparrow, S., Wang, J., Lord, C., Dawson, G., Fombonne, E., Loveland, K., Mesibov, G., \& Schopler, E. (1998). The Vineland Adaptive Behavior Scales: Supplementary Norms for Individuals with Autism. Journal of Autism and Developmental Disorders, 28.287-302.

Carr, E., \& Durand, V. (1985). Reducing behavior problems through functional communication training. Journal of Applied Behavior Analysis, 18, 111-126.

Carr, E., McLaughlin, D., Giacobbe-Grieco, T., \& Smith, C. (2003). Using mood ratings and mood induction in assessment and intervention for severe problem behaviour. American Journal on Mental Retardation, 108, 32-55.

Christie, R., Bay, C., Kaufman, I. A., Bakay, B., Borden, M. and Nyhan, W. L. (1982). Lesch-Nyhan disease: clinical experience with nineteen patients. Developmental Medicine and Child Neurology, 24, 293-306.

Cohen, J. (1960). A coefficient of agreement for nominal scales. Educational and Psychological Measurement, 20, 37-46.

Colley, A., Leversha, M., Voullaire, L., \& Rogers, J. (1990). Five cases demonstrating the distinctive behavioural features of chromosome deletion 17 (p11.2 p11.2) (Smith-Magenis syndrome). Journal of Paediatric Child Health, 26, 17-21.

Crumley, F. (1998). Smith-Magenis syndrome. Journal of American Academy. Child and Adolescent Psychiatry, 37, 11.

De Leersnyder, H., Bresson, de Blois, M., Souberbielle, J-C., Mogenet, A., DelhotalLanders, B., Salefranque, F., \& Munnich, A. (2003). Beta-adrenergic antagonists and 
melatonin reset the clock and restore sleep in a circadian disorder: Smith-Magenis syndrome. Journal Medical Genetics, 40, 74-78.

De Leersnyder, de Blois, M., Munnich, A., Nolen, M.C., SaleFranque, F. \& Claustrat, B. (1999). Smith-Magenis Syndrome: an original sleep disturbance in children's sleep. Research Online, 2, 494.

De Leersnyder, H., de Blois, M., Claustrat, B., Romana, S., Albrecht, U., von KleistRetzow, J., Delobel, B., Viot, G., Lyonnet, S., Vekemans, M., \& Munnich, A. (2001a). Inversion of the circadian rhythm of melatonin in the Smith-Magenis syndrome. The Journal of Pediatrics, 139, 111-116.

De Leersynder, H., de Blois, M., Vekemans, M., Sidi, D., Villian, E., Kindermans, C., \& Munnich, A. (2001b). B1-adrenergic antagonists improve sleep and behavioural disturbances in a circadian disorder, Smith-Magenis syndrome. Journal of Medical Genetics, 38, 586-590.

Dykens, E. (1995). Measuring behavioural phenotypes: Provocations from the "New Genetics." American Journal on Mental Retardation, 99, 522-532.

Dykens, E., Finucane, B., \& Gayley, C. (1997). Brief Report: Cognitive and Behavioural Profiles in Persons with Smith-Magenis Syndrome. Journal of Autism and Developmental Disorders, 27, 203-211.

Dykens, E., \& Smith, A. (1998). Distinctiveness and correlates of maladaptive behaviour in children and adolescents with Smith-Magenis syndrome. Journal of Intellectual Disability Research, 42, 481-489.

Finucane, B., Dirrigl, K., \& Simon, F. (2001). Characterisation of self-injurious behaviours in children and adults with Smith-Magenis syndrome. American Journal of Mental Retardation, 106, 52-58.

Finucane, B., Konar, D., Haas-Givler, B., Kurtz, M., \& Scott, C. (1993). Self-hugging as a diagnostic marker in Smith-Magenis Syndrome. American Journal of Human Genetics, 53, 431.

Finucane, B., Konar, D., Haas-Givler, B., Kurtz, M., \& Scott, C. (1994). The Spasmodic Upper-body Squeeze: a Characteristic Behaviour in Smith-Magenis Syndrome.

Developmental Medicine and Child Neurology, 36, 70-83.

Finucane, B., Kurtz, M., Babu, R., \& Scott, C. (1993). Mosaicism for Deletion 17p11.2 in a boy with the Smith-Magenis syndrome. American Journal of Medical Genetics, 45, 447449.

Fleiss, J. (1981). Statistical Methods for rates and proportions ( $2^{\text {nd }}$ ed.). New York: John Wiley.

Greenberg, F., Guzzetta, R., Montes de Oca-Luna, R., Magenis, Smith, A.C., Richter, S.F., Kondo, I., Dobyns, W., Patel, P., \& Lupski, J.R. (1991). Molecular analysis of the Smith 
Magenis Syndrome: a possible contiguous-gene syndrome associated with del (17) (p11.2). American Journal of Human Genetics, 49, 1207-1218.

Grossman, H. (Ed.). (1983). Classification in Mental Retardation. Washington DC: American Association on Mental Deficiency.

Hall, S. \& Oliver, C. (1992). Differential effects of severe self-injurious behaviour on the behaviour of others, Behavioural Psychotherapy, 20, 355-365.

Hall, S., Oliver, C., \& Murphy, G. (2001a). Early development of self-injurious behaviour: An Empirical Study. American Journal of Mental Retardation, 106, 189-199.

Hall, S., Oliver, C., \& Murphy, G. (2001b). Self-injurious behaviour in young children with Lesch-Nyhan syndrome. Developmental Medicine and Child Neurology, 43, 745-749.

Harding, J., Wacker, D., Berg, W., Barretto, A., \& Ringdahl, J. (2005). Evaluation of Relations Between Specific Antecedent Stimuli and Self-Injury During Functional Analysis Conditions. American Journal on Mental Retardation, 110, 205-215.

Haas-Givler, B. (1994). Educational implications and behavioural concerns of SmithMagenis syndrome - From the teachers' perspective. Spectrum, 1, 36-38.

Haas-Givler, B. \& Finucane, B. (1995). What's a teacher to do: Classroom strategies that enhance learning for children with Smith-Magenis syndrome. Spectrum, 2.

Horsler, K., \& Oliver, C. (In press). Environmental influences on the behavioural phenotype of Angelman syndrome. American Journal of Mental Retardation.

Hyman, P., Oliver, C., \& Hall, S. (2002). Self-injurious behaviour, self-restraint, and compulsive behaviours in Cornelia de Lange syndrome. American Journal on Mental Retardation, 107, 146-154.

Iwata, B., Dorsey, M., Slifer, K., Bauman, K., \& Richman, G. (1982). Towards a functional analysis of self-injury. Analysis and Intervention in Developmental Disabilities, 2, 3-20.

Juyal, R., Figuera, L.., Hauge, X., Elsea, S., Lupski, J., Greenberg, F., Baldini, A., \& Patal, P. (1996). Molecular analyses of $17 \mathrm{p} 11.2$ deletions in 62 Smith-Magenis syndrome patients. American Journal of Human Genetics, 58, 998-1007.

King, R., Nixon, J., Beatty, K., Szalajko, J., Marwick, L., \& Prescott, H. (2004). Posttraumatic stress disorder and phenotype behaviour in an individual with Smith-Magenis syndrome: A multimodal approach. Mental health aspects of developmental disabilities, 7 , 131-141.

Lesch, M., \& Nyhan, W. (1964). A familiar disorder of uric acid metabolism and centre nervous system function. American Journal of Medicine, 36, 561-570. 
Lockwood, D., Hecht, F., Dowman, C., Hecht, B., Rizkallah, T., Goodwin, T., \& Allanson, J. (1988). Chromosome subband 17p11.2 deletion: a minute deletion syndrome. Journal of Medical Genetics, 25, 732-737.

Madduri, N., Turcich, M., Lupski, J., \& Potocki, L. (2002). Low adaptive behaviour and cognitive functioning in patients with Smith-Magenis syndrome [del(17)(p11.2p11.2)]. American Journal of Human Genetics, 71 (suppl): A109.

Martin, N., Oliver, C., \& Hall, S. (2001). Obswin: software for the collection and analysis of observational data. University of Birmingham: Birmingham, UK..

McElwee, C., \& Bernard, S. (2002). Genetic syndromes and mental retardation. Current Opinion in Psychiatry, 15, 469-475.

Moss, J., Oliver, C., Arron, K., Burbidge, C. and Berg, K. (In review). The Prevalence and Phenomenology of Repetitive Behaviour in Genetic Syndromes. Psychological Medicine.

Moss, J., Oliver, C., Hall, S., Arron, K., Sloneem, J., \& Petty, J. (2005). The association between environmental events and self-injurious behaviour in Cornelia de Lange syndrome. Journal of Intellectual Disability Research, 49, 269-277.

Moncla, A., Livet, M., Auger, M., Mattei, J., Mattei M., \& Giraud, F. (1991). Journal of Medical Genetics, 28, 9, 627-632.

Oliver, C. (1993). Self-injurious behaviour: From response to strategy. In C. Kiernan (Ed.), Challenging behaviour and learning disabilities: Research to practice?: Implications of research on the challenging behaviour of people with learning disabilities (pp.135-188). Clevedon, Bristol: BILD Publications.

Oliver, C. (1995). Annotation: Self-injurious behaviour in children with learning disabilities: Recent advances in assessment and intervention. Journal of Child Psychology and Psychiatry, 36, 909-927.

Oliver, C., Berg, K., Burbidge, C, Arron K. and. Moss, J. (In review). Delineation of behavioural phenotypes in genetic syndromes. 1. Comparison of autism spectrum disorder, affect and hyperactivity. British Journal of Psychiatry

Oliver, C., Demetriades, \& Hall, S. (2002). Effects of Environmental Events on Smiling and Laughing in Angelman Syndrome. American Journal on Mental Retardation, 107, 194200.

Oliver, C., Hall, S., \& Murphy, G. (2005). The early development of self-injurious behaviour: evaluating the role of social reinforcement. Journal of Intellectual Disability Research, 49, 591-599.

Oliver, C., Hall, S., \& Nixon, J. (1999). The molecular and molar analysis of communicative and problem behaviours. Research in Developmental Disabilities, 20, $197-$ 213.

Oliver, C., Horsler, K., Berg, K., Bellamy, G., Dick, K. \& Griffiths, E. (2007). Genomic 
imprinting and the expression of affect in Angelman syndrome. What's in the smile? Journal of Child Psychology and Psychiatry, 48, 571-579.

Oliver, C., Murphy, G., Crayton, L., \& Corbett, J. (1993). Self-injurious behaviour in Rett syndrome: interactions between features of Rett syndrome and operant conditioning. Journal of Autism Development Disorder, 23, 91-109.

Potocki, L., Shaw, C., Stankiewicz, P., \& Lupski, J. (2003). Variability in clinical phenotype despite common chromosomal deletion in Smith-Magenis syndrome [del (17) (p11.2p11.2)]. Genetics in Medicine, 5, 430-434.

Smith, A., Dykens, E., \& Greenberg, F. (1998a). Behavioural Phenotype of Smith-Magenis Syndrome (del 17p11.2). American Journal of Medical Genetics (Neuropsychiatric Genetics), 81, 179-185.

Smith, A., Dykens, E., \& Greenberg, F. (1998b). Sleep Disturbance in Smith-Magenis Syndrome (del 17 p.11.2). American Journal of Medical Genetics (Neuropsychiatric Genetics) 81, 186-191.

Smith, A., McGavran, L., Robinson, J., Waldstein, G., Macfariane, J., Zonona, J., Reiss, J., Lahr, M., Allen, L., \& Magenis, E. (1986). Interstitial Deletion of (17) (p11.2p11.2) in nine patients. American Journal of Medical Genetics, 24, 393-414.

Spencer, M. (2003). He's not drowning. He's waving. England: Solomanwhite Publications.

Stratton, R., Dobyns, W., Greenberg, F., DeSana, J., Moore, C., Fidone, G., Runge, G., Feldman, P., Sekhon, G., Pauli, R., \& Ledbetter, D. (1986). Interstitial Deletion of (17) (p11.2p11.2): Reports of Six Additional Patients with a New Chromosome Deletion Syndrome. American Journal of Medical Genetics, 24, 421-432.

Shelley, B. P. Robertson, M. M. \& Turk, J. (2007). An individual with Gilles de la Tourette syndrome and Smith-Magenis microdeletion syndrome: is chromosome 17p11.2 a candidate region for Tourette syndrome putative susceptibility genes? Journal of Intellectual Disability Research, 51, 620-624.

Taylor and Oliver (In preparation). The behavioural phenotype of Smith-Magenis syndrome.

Udwin, O., Webber, C., \& Horn, I. (2001). Abilities and attainment in Smith-Magenis syndrome. Developmental Medicine and Child Neurology, 43, 823-828.

Willekens, D., Cock, P., \& Fryns, J. (2000). Three young children with Smith-Magenis Syndrome: Their distinct, recognisable behavioural phenotype as the most important clinical symptoms. Genetic Counselling, 11, 103-110.

Yoder, P., \& Feurer, I. (2000). Quantifying the magnitude of sequential association between events and behaviors. In T. Thompson, D. Felce, \& F. Symons (Eds.), Behavior 
observation: Technology and applications in developmental disabilities (p. 317-334). Baltimore: Brookes. 


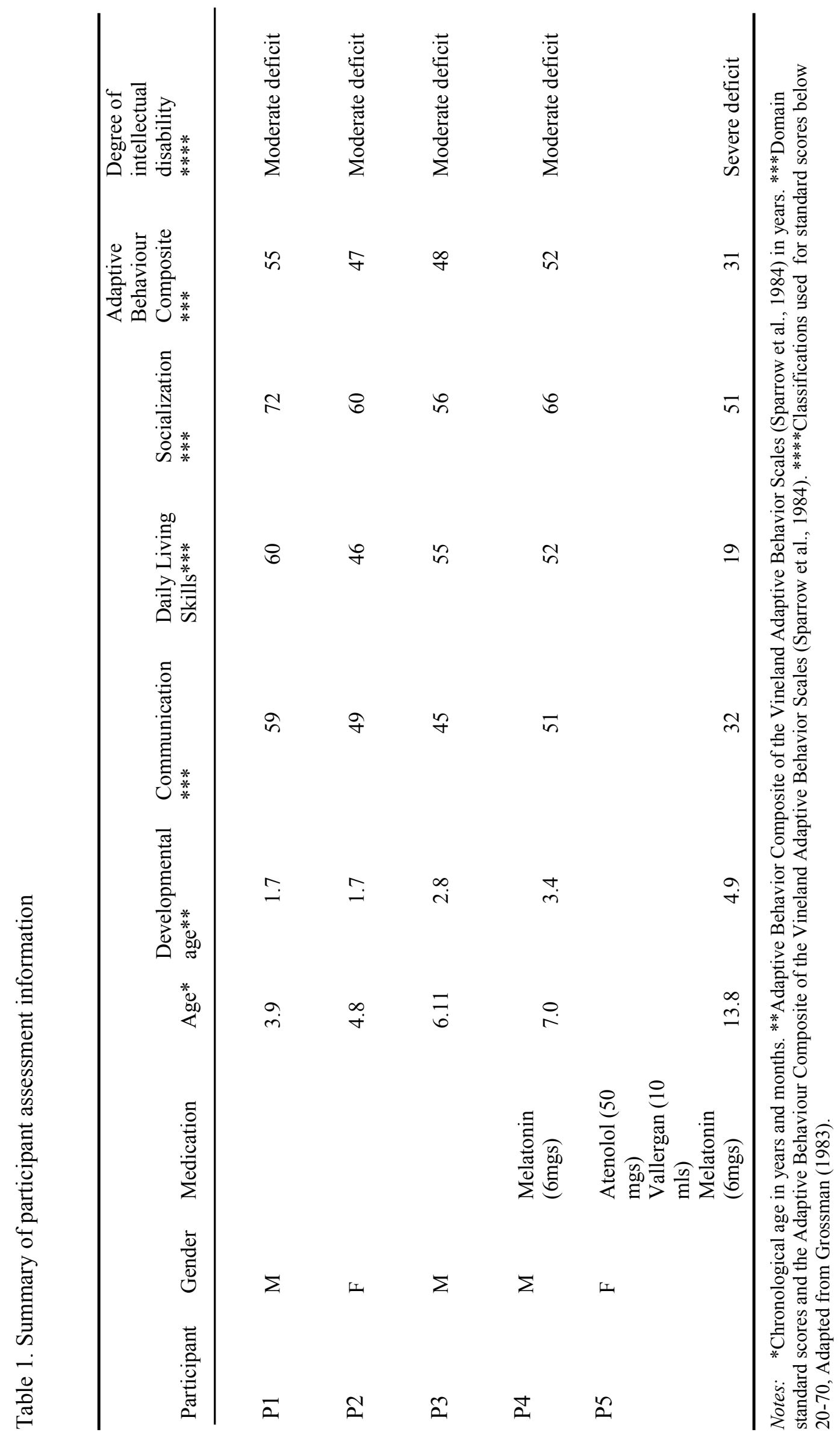


Table 2: $\quad$ Percentage duration for participants' problem behaviours

\begin{tabular}{|c|c|c|c|c|c|}
\hline \multirow[t]{2}{*}{ Behaviour } & \multicolumn{5}{|c|}{ Participant } \\
\hline & P1 & P2 & P3 & P4 & P5 \\
\hline & 12.4 & 12.4 & 9.5 & 9.3 & 10.2 \\
\hline Total observation time & hours & hours & hours & hours & hours \\
\hline SELF INJURIOUS BEHAVIOUR & 10.26 & 7.37 & 3.17 & 3.05 & 1.07 \\
\hline Biting self & 7.93 & 6.75 & 2.3 & 1.31 & .52 \\
\hline Head Banging & 0 & .11 & .19 & 1.65 & 0 \\
\hline Nail and skin picking & .03 & .2 & .09 & .06 & .19 \\
\hline Self slapping & .51 & .32 & .07 & .16 & 0 \\
\hline Poke eye & 2.6 & 0 & 0 & 0 & .05 \\
\hline Insert fingers up nose & 0 & 0 & .55 & 0 & .31 \\
\hline \multicolumn{6}{|l|}{ AGGRESSIVE/DISRUPTIVE } \\
\hline OUTBURST & 5.81 & 3.08 & 3.76 & 4.18 & .17 \\
\hline Bang object & 2.81 & 1.23 & .55 & 2.09 & .15 \\
\hline Kicking & 2.94 & 1.12 & 0 & 1.21 & 0 \\
\hline Push away & .02 & 0 & .26 & .02 & 0 \\
\hline Strike others & .28 & .75 & .27 & .01 & .01 \\
\hline Spits & 0 & 0 & 0 & .86 & 0 \\
\hline
\end{tabular}


Table 3: Yule's Q values to assess the co-occurrence in 10 second time windows for problem behaviours and environmental events

\begin{tabular}{lrr}
\hline & $\begin{array}{c}\text { Self- } \\
\text { Injurious } \\
\text { Behaviour }\end{array}$ & $\begin{array}{r}\text { Aggressive/ } \\
\text { Disruptive } \\
\text { Outbursts }\end{array}$ \\
\hline P1 & .02 & -.11 \\
Attention & -.33 & .19 \\
Demand & & \\
P2 & .13 & -.07 \\
Attention & .03 & -.27 \\
Demand & & \\
P3 & -.02 & -.31 \\
Attention & -.13 & .26 \\
Demand & & \\
P4 & & -.52 \\
Attention & .52 & .16 \\
Demand & .25 & \\
P5 & & -.10 \\
Attention & & \\
Demand & -.44 & \\
\hline
\end{tabular}

Notes: Significant positive values (>.30) are in bold text. Significant negative values are in italics and bold. 


\section{Legend for Figure 1.}

Figure 1. Unconditional probability of social events and the probability of social events given the onset of an episode of problem behaviour (conditional probability) for 120 s prior to and following the onset of an episode of challenging behaviour. The shaded area indicates that Yule's Q, employed as an index of the difference between unconditional and conditional probabilities, exceeds .3. The vertical line at lag 0 indicates the point of onset for the problem behaviour. 